\title{
La participación de los stakeholders en los procesos de gobernanza: mito y realidad
}

\section{Participation of Stakeholders in Governance Processes: Myth and Reality}

\author{
José A. Estévez Araújo \\ Joan Ramos Toledano ${ }^{1}$
}

\begin{abstract}
Resumen: El presente artículo tiene por objeto analizar los niveles de participación de los stakeholders (interesados) en la toma de decisiones relativas a las políticas de lucha contra la pobreza y la exclusión social en el marco de la Unión Europea. Esta toma de decisiones se articula a través de mecanismos de gobernanza, los cuales son considerados legítimos en la medida en que permiten a los interesados participar activamente en la consideración, adopción e implementación de las políticas. Por ello aquí se pretende determinar cuál es la participación real de los stakeholders en los mecanismos de gobernanza en la UE. Se propone una escala en la que se establecen cuatro niveles distintos de "participación", desde la mera información hasta el empoderamiento para poder implementar las decisiones tomadas. Los resultados que se exponen en el texto son fruto de diversas entrevistas, cuestionarios y conversaciones con diferentes representantes, tanto de organismos públicos estatales (de España y otros países europeos) como de entidades del Tercer Sector ${ }^{2}$. La investigación permite concluir que existe una importante brecha entre el discurso oficial acerca de la participación y el modo como se adoptan e implementan realmente las decisiones en el ámbito del llamado "Semestre Europeo".
\end{abstract}

Palabras clave: Gobernanza, participación, stakeholders, Unión Europea. Semestre Europeo, pobreza.

\begin{abstract}
The purpose of this article is to analyze the levels of participation of stakeholders in decision-making regarding policies to combat poverty and social exclusion within the framework of the European Union. This decision-making is articulated through governance mechanisms, that are legitimated to the extent that they allow the stakeholders to participate actively in the consideration, adoption and implementation of policies. The main objective is to analyze the real participation of stakeholders in the governance mechanisms in the EU. To this end, we propose a scale in which four various levels of "participation" are

\footnotetext{
1 José A. Estévez Araújo es Catedrático de Filosofía del Derecho de la Universitat de Barcelona. Joan Ramos toledano es Doctor en Ciudadanía y Derechos humanos y miembro del Equipo de Investigación consolidado de Filosofía del Derecho, Moral y Política de la misma Universidad.

${ }^{2}$ La "Taula d'Entitats del Tercer Sector" (institución que representa el conjunto de las entidades sociales catalanas; www.tercersector.cat) define el "Tercer Sector Social" como el conjunto de entidades privadas sin ánimo de lucro que trabajan por la inclusión y la cohesión social, con especial atención a los colectivos vulnerables de la sociedad. El concepto "tercer sector" define las entidades no gubernamentales y no lucrativas en contraposición al "primer sector" o "sector público" (gubernamental y no lucrativo) y al "segundo sector" o sector de la empresa privada (no gubernamental y lucrativo).
} 
established, from mere information to empowerment in order to implement the decisions and policies. The outcome is the result of various interviews, questionnaires and conversations with different representatives, both from state public bodies (from Spain and other European countries) and from Third Sector entities ${ }^{3}$. The research allows us to conclude that there is an important gap between the theoretical assumptions of participation and the reality in the adoption and implementation of decisions in the framework of the European Semester.

Keywords: Governance; Participation; Stakeholders; European Union; European Semester; Poverty

\section{INTRODUCCIÓN}

El presente artículo tiene por objeto analizar el nivel de legitimidad de los procesos de toma de decisiones relativas a las políticas de lucha contra la pobreza y la exclusión social en el marco de la Unión Europea ${ }^{4}$. Estas decisiones se han adoptado mediante procesos de gobernanza. El uso del término "gobernanza" se ha generalizado, revistiéndose por ello de un alto grado de ambigüedad. Por ello dedicaremos el primer apartado del texto a explicitar el significado de este concepto, tanto en su sentido amplio como en un sentido más estricto. Explicaremos también qué especie o tipo de gobernanza se ha utilizado en el diseño de las políticas contra la pobreza en la UE.

En el segundo apartado expondremos la situación de la pobreza en Europa, analizando las causas y los niveles de la misma. También discutiremos el sentido de las políticas de lucha contra la pobreza puestas en marcha en Europa desde el año 2010 y explicaremos los motivos de su fracaso. Estas dos secciones confluirán en la tercera en la que se expondrán los resultados de nuestra investigación acerca de la posibilidad real de participación que han tenido los stakeholders o interesados (personas pobres y organizaciones sociales) en el diseño de dichas políticas. Nos centraremos especialmente en el caso español.

\footnotetext{
3 The "Taula d'Entitats del Tercer Sector" (institution that represents all the Catalan social entities, www.tercersector.cat) defines the "Third Social Sector" as the set of non-profit private entities that work for inclusion and social cohesion, with special attention to vulnerable groups in society. The concept "third sector" defines non-governmental and non-profit entities as opposed to the "first sector" or "public sector" (governmental and non-profit) and the "second sector" or sector of private companies (non-governmental and profit).

${ }^{4}$ Este texto es fruto de la investigación en el marco del proyecto "La legitimidad de la gobernanza en tiempos de crisis: análisis del funcionamiento del Método Abierto de Coordinación (MAC) en España, Portugal e Italia de 2010 a 2015" (DER2014-53891-P, financiado por el Ministerio de Economía y Competitividad), cuyo objetivo era analizar la legitimidad de la gobernanza en la coordinación de las políticas de lucha contra la pobreza en la Unión Europea.
} 


\section{¿QUÉ ES LA GOBERNANZA?}

El término "gobernanza" puede ser utilizado en un sentido muy amplio o de forma más estricta. En sentido amplio se refiere a la dirección política o a la regulación de las políticas públicas. Se podría traducir por "gobernación" en el sentido de acción de gobernar. La palabra "gobierno" tiene ya ese significado, pero puede referirse también a la institución específica a la que se atribuye el poder ejecutivo, por lo que resulta ambigua. La gobernanza, en este sentido amplio, incluye todos los actores, estructuras y procesos que intervienen en esa regulación general de los asuntos de interés público. Por ejemplo, si hablamos de la gobernanza europea de la política económica, estamos usando el término en ese sentido amplio.

La palabra "gobernanza" puede utilizarse en un sentido más estricto. Se refiere, entonces, a una transformación en los métodos de regulación de las políticas públicas, a un cambio en los actores que intervienen en la misma y a una modificación en la forma de relacionarse entre ellos.

La regulación tradicional de los asuntos públicos estaba confiada al Estado. Este tenía una estructura jerárquica. Estaba organizado de acuerdo con el modelo burocrático descrito por Max Weber y conformaba un sistema de «ordeno y mando», que utilizaba medidas coactivas para alcanzar sus objetivos.

A partir de las crisis políticas, sociales y económicas de finales de los sesenta y principios de los setenta, se empezó a poner en cuestión la capacidad regulatoria del Estado. El texto de Huntinton, Crozier y Watanukis sobre la crisis de la democracia es buena prueba de ello (Crozier, Huntington and Watanuki, 1975). El subtítulo del libro era "Report on the governability of democracies to the Trilateral Commission." Apareció en escena, así, la palabra "gobernabilidad". Ese término se refería a la posibilidad/capacidad de una dirección política efectiva de los asuntos públicos. Los autores sostenían que las democracias existentes eran ingobernables.

En las dos décadas siguientes se desarrollan nuevas formas de dirección de las políticas públicas, que involucran directamente a actores privados, como las empresas o las organizaciones de la sociedad civil. La relación entre las diversas partes implicadas deja de ser del tipo ordeno y mando para adoptar formas más cooperativas y utilizar mecanismos de negociación. En ocasiones, el Estado se limita a establecer unos espacios de encuentro para que los afectados discutan entre sí, de cara a identificar los problemas y determinar la forma en que deben ser regulados. ${ }^{5}$ Así, por ejemplo, en el ámbito de las políticas ecológicas el Estado puede crear un espacio de diálogo entre los interesados (empresas, vecinos, movimientos ecologistas...) y limitarse a poner un marchamo oficial a lo que esos actores acuerden.

La estructura de los órganos públicos también se transforma, perdiendo, al menos en parte, su carácter jerárquico. En el seno de la Administración aparecen procesos de cooperación negociada. Unos tienen carácter horizontal y se dan entre

${ }^{5}$ Es lo que en el ámbito de la Sociología jurídica se denomina "procedimentalización del Derecho" (Estévez Araujo, 2006). 
instancias del mismo nivel, por ejemplo, por medio de comisiones interministeriales. Otras tienen carácter vertical y se dan entre instancias de distintos niveles. En la Unión Europea se habla de «gobernanza multinivel» para referirse a las diferentes escalas de los organismos públicos: europea, estatal, regional y municipal. La relación entre estos tres ámbitos no es necesariamente jerárquica, sino que muchas decisiones se adoptan mediante la negociación entre órganos de dos, o de los tres niveles.

Las estructuras de dirección política han empezado a utilizar cada vez con mayor frecuencia métodos no coactivos de ejecución de las regulaciones, estableciendo incentivos en lugar de sanciones. Por ejemplo, una empresa puede no estar obligada a cumplir una determinada normativa, pero solo podrá obtener contratos públicos si la cumple, o bien obtendrá una certificación que mejorará su imagen de cara a los consumidores (p. ej. que utiliza productos ecológicos). También se utilizan mecanismos de emulación competitiva, como los rankings entre Universidades, o formas de «señalar con el dedo» para provocar «vergüenza», como cuando la Comisión Europea hace una Recomendación en la que critica la actuación de un determinado Estado.

En síntesis, cuando hablamos de gobernanza en este sentido más estricto, nos estamos refiriendo a un sistema de regulación de las políticas públicas que no está centrado exclusivamente en el Estado, sino en el que participan otros agentes; a unos mecanismos de decisión que no se basan en la jerarquía, sino en la cooperación negociada, minando la estructura burocrática de la administración pública e instaurando instrumentos de coordinación horizontales y verticales; y a una regulación que no utiliza solo sanciones, sino crecientemente estímulos de carácter económico o de prestigio. $^{6}$

Hay diversas especies de gobernanza entendida esta en sentido estricto. Cada una de ellas gradúa y combina de manera diferente los elementos señalados. En este texto, nos ocuparemos de una de esas especies: el llamado «experimentalismo democrático». Su denominación señala por sí misma los dos componentes fundamentales de esta forma de gobernanza: uno es la experimentación, el otro es la democracia. "Experimentar" significa utilizar el método del ensayo y el error, con el objetivo de crear oportunidades para encontrar soluciones imaginativas y corregir a tiempo errores de planteamiento. El otro elemento es el ingrediente "democrático". Esta modalidad de gobernanza pone especial énfasis en la participación directa de los stakeholders o afectados. Estos no deberían limitarse a negociar en función de sus intereses, sino que tendrían que ser capaces de adoptar una disposición a dialogar para encontrar la mejor solución posible a los problemas prácticos que se les plantean. La participación es, pues, un elemento nuclear de la legitimidad de las decisiones adoptadas por medio de este tipo de gobernanza (Mercado Pacheco, 2012).

\footnotetext{
${ }^{6}$ El autor que introdujo la temática de la gobernanza en España fue Joan Prats (Prats Català, 2005).
} 


\section{LA POBREZA EN LA UE}

Es muy difícil entender qué significa la pobreza para alguien que no esté en esa situación, pues ser pobre tiene consecuencias sociales, culturales, laborales, sanitarias, psicológicas..., además de económicas. Cuando los indigentes hablan de su situación, dicen que viven en un estado de emergencia permanente. La pobreza no es una ocasional falta de dinero. Es una lucha cotidiana para poder sobrevivir, uno mismo y las personas a cargo de uno. Los pobres perciben el desprecio que los otros sienten por ellos y eso les provoca una profunda humillación. No pueden alimentar adecuadamente a sus hijos ni hacerles regalos de navidad como otros padres a sus niños. Tienen vedado el acceso a muchos bienes culturales y a espacios de ocio. Escuchar a quienes viven en la pobreza debería ser una prioridad absoluta para quienes tienen la responsabilidad de combatirla.

La Unión Europea es una región muy rica en términos de su PIB. Pero en ella viven un número de personas pobres que no ha hecho sino aumentar desde los años setenta del pasado siglo. En el año 2016 había casi 120 millones de personas en situación de pobreza en la UE, según los datos de Eurostat. De ellos, 75 millones eran habitantes de la zona euro.

La incidencia de la pobreza es muy diferente en los distintos países de la Unión. España tenía más de un $27 \%$ de pobres en 2016, Italia un 30\% y Grecia más de un 35\%. Sin embargo, Dinamarca y Holanda oscilaban en torno a un 15\% de personas en situación de pobreza. Si nos vamos a los casos más extremos, Bulgaria y Rumanía tenían en torno a un $40 \%$ de personas en ese estado, pero Bulgaria había conseguido sacar a un 10\% de su población de la pobreza desde 2010 , cuando había un $50 \%$ de personas pobres en el país. Las diferencias entre las regiones de un mismo país pueden ser incluso más acusadas que las diferencias entre Estados. $^{8}$

Estas cifras se refieren a una pobreza relativa, no absoluta. Según la ONU, están en situación de pobreza extrema quienes viven con menos de 1,25 dólares al día. La pobreza relativa se determina en función de los estándares de vida de cada país, como, por ejemplo, según los ingresos en relación con la renta media. Por tanto, no es lo mismo ser relativamente pobre en un país rico o serlo en uno pobre. La pobreza en un país pobre puede significar no tener acceso a los bienes necesarios para satisfacer las necesidades más básicas. En un país rico, con una distribución relativamente equitativa de la riqueza, sigue siendo una situación con terribles consecuencias, pero no una lucha cotidiana a vida o muerte.

\footnotetext{
${ }^{7}$ Hay un libro editado por la Plataforma Europea de Lucha contra la pobreza (EPAP) en el que se recogen testimonios de personas pobres acerca de su situación (EAPN, 2014).

${ }^{8}$ Los datos sobre la evolución de la pobreza en Europa pueden encontrarse en la siguiente página de Eurostat: http://ec.europa.eu/eurostat/tgm/refreshTableAction.do?tab=table\&plugin=1\&pcode=t2020_50\&language $=\mathrm{en}$
} 
La Unión Europea diseñó un programa para la presente década llamado “Estrategia Europa 2020". Se puso en marcha el año 2010 y se proponía el objetivo de reducir la pobreza en 20 millones de personas para el año $2020{ }^{9}$

El criterio para medir la pobreza no es uniforme en todos los países de la UE. La Estrategia Europa 2020 no ha establecido tampoco un baremo único, sino que señala que hay tres maneras de definir la pobreza. La primera se refiere al nivel de ingresos. Percibir una renta por debajo del $60 \%$ de la media es ser pobre. La segunda se centra en la privación material severa: se establecen 9 ítems que se consideran básicos (p. ej. tener una lavadora) y quien carezca de cuatro o más de ellos es considerado pobre. La tercera se refiere a la intensidad del trabajo: si el número de adultos residentes en un hogar han trabajado menos del $20 \%$ de su potencial tiempo de empleo, entonces ese hogar es pobre. Además, países como Alemania, Gran Bretaña o Suecia utilizan criterios diferentes a los tres establecidos en la Estrategia 2020. Alemania mide la pobreza en función de los parados de larga duración, Suecia combina los parados de larga duración y los enfermos crónicos y Gran Bretaña se basa en la pobreza infantil (Copeland et al., 2014).

Los diferentes Estados de la UE han podido elegir uno, dos o los tres criterios y seleccionan el o los que les resultan más favorables. Además, cada uno de ellos ha cuantificado independientemente el objetivo a alcanzar, es decir, a cuántas personas pretende sacar de la pobreza en esta década. Hay países, como Francia o Dinamarca, que han reducido ligeramente la tasa de pobreza y otros que lo han hecho de forma más acusada, como es el caso de Bulgaria, ya mencionado, o el de las repúblicas bálticas. Los países más afectados por la crisis de la Eurozona, como Grecia, Italia o España, han visto aumentar la tasa de personas en situación de pobreza. De estos, únicamente en Portugal ha disminuido ligeramente esa proporción de pobres entre 2010 y 2016.

Se puede afirmar ya que el objetivo de la Estrategia 2020 no se va a alcanzar. Ahora hay más pobres en la Unión Europea que en 2010. Solo en la Eurozona se contabiliza un aumento de aproximadamente tres millones.

La Unión Europea viene desarrollando iniciativas de lucha contra la pobreza desde los años setenta del siglo pasado. Pero los organismos europeos no tienen propiamente poder en esta materia. La tarea de luchar contra la pobreza es competencia exclusiva de los Estados miembros. La UE solo puede realizar acciones de apoyo, orientación y coordinación.

Así, por ejemplo, 2010 fue proclamado por la Comisión Europea como "año de lucha contra la pobreza". Los fondos comunitarios dedicados a esta iniciativa fueron ridículamente bajos: 17,5 millones de euros para los 27 países de la Unión. Con ellos se financiaron actuaciones tan peculiares como una gira de jóvenes artistas en Portugal para sensibilizar a la población acerca del problema de la pobreza, o una serie de programas de radio en Holanda para que las personas po-

\footnotetext{
9 "El número de europeos que viven por debajo de los umbrales nacionales de pobreza debería reducirse en un $25 \%$, rescatando así a más de 20 millones de personas de la pobreza." V. http://eur-lex.europa.eu/legalcontent/ES/TXT/?uri=celex:52010DC2020
} 
bres explicaran sus experiencias. Fueron acciones de propaganda que no tuvieron demasiado éxito ni lograron aumentar la implicación de los Estados en el problema. Buena muestra de ello es que el número de pobres en la UE aumentó durante ese año.

La estrategia Europa 2020 supuso un avance. Estableció objetivos cuantificados, entre los cuales el de sacar a veinte millones de personas de la pobreza, como se ha dicho. No obstante, la competencia en esta materia siguió siendo exclusivamente de los Estados. Algunos de ellos han visto la fijación de esta cifra como una intrusión en materias sobre las que tienen titularidad exclusiva. Es el caso de Alemania, Suecia o el Reino Unido, que, como se vio, no aceptaron ninguno de los criterios de pobreza establecidos por la Comisión.

Aunque la UE no tenga competencia en materia de lucha contra la pobreza, su política económica sí que afecta a la evolución de la misma. Las medidas de austeridad impuestas tras la crisis de la Eurozona no favorecen el desarrollo, sino que lo obstaculizan. Las exigencias europeas de equilibrio presupuestario obligan a los Estados a realizar recortes y estos están afectando especialmente a las políticas sociales. El aumento de la deuda pública como consecuencia de los rescates bancarios empeoró enormemente la situación presupuestaria de países como España. El Estado español pasó de tener una deuda pública equivalente al $35 \%$ del PIB en 2007 a soportar en 2014 una deuda equivalente al 100\% del mismo, porcentaje que no ha disminuido desde entonces. Durante estos últimos años la prioridad de la política económica española ha sido pagar a los bancos la deuda contraída para rescatarlos, que fue sancionada por la reforma del artículo 135 de la Constitución. ${ }^{10}$

La política económica y financiera es, pues, la principal causa del aumento de la pobreza en Europa. Las políticas dirigidas a combatirla se han mostrado inadecuadas e insuficientes. La causa de esto es la filosofía de la lucha contra la pobreza que presuponen.

Uno de los conceptos clave que subyace a la estrategia de lucha contra la pobreza es el de "crecimiento inclusivo". Esta expresión significa que las políticas contra la pobreza se centran en la entrada o vuelta al mercado de trabajo. Parece que ahora lo único necesario para disminuir el número de pobres es incrementar el crecimiento y el empleo (Peña-Casas, 2012). Desde luego, es cierto que una de las principales causas de la pobreza es el desempleo. Sin embargo, no es la única. Además, conseguir un trabajo no necesariamente saca a las personas de la pobreza.

Una de las razones de la existencia de "trabajadores pobres" es que la Estrategia Europa 2020 no se preocupa de la calidad del empleo sino solo de su productividad. Las políticas laborales en la mayoría de los países de la UE se han centrado en la "flexibilidad", aunque en ocasiones la hayan vestido de "flexiguridad". Se han multiplicado las formas de trabajo precario y los salarios han disminuido (Arriola, 2014). Esto demuestra que el aumento del empleo sin incremento de las rentas percibidas por los trabajadores no es suficiente para disminuir la pobreza.

${ }^{10}$ Los datos sobre la evolución de la deuda pública en relación con el PIB relativos a los diferentes países de la UE están disponibles en Eurostat. 
El caso de Alemania pone de manifiesto una gran discordancia entre el nivel de desempleo y el de pobreza (5\% versus casi $17 \%)$. Esto se debe a que muchos trabajadores alemanes tienen un salario que no les permite dejar de ser pobres como consecuencia de las reformas laborales regresivas llevadas a cabo en los 2000. Fuera de la Eurozona, ocurre lo mismo en Gran Bretaña, donde el desequilibrio es aún mayor: la tasa de pobreza es cuatro veces mayor que la de desempleo. En Holanda, el porcentaje de pobres dobla al de parados. Sin embargo, en el caso de España hay un $17 \%$ de desempleados y un $22 \%$ de pobres, lo que supone un mayor grado de correspondencia entre desempleo y pobreza (Eurostat). Estos datos permiten inferir que los países con bajos índices de paro y altos índices de pobreza han "precarizado" a sus trabajadores.

La pobreza es un fenómeno multidimensional. No responde a una única causa. Personas en igual situación según los indicadores seleccionados, pueden vivir en condiciones muy diferentes. Una madre soltera con hijos a su cargo estará en situación peor que una pareja sin hijos, aunque su nivel de ingresos sea el mismo. Incluso una combinación de los tres indicadores puede encubrir muchas diferencias. Dos familias pueden tener el mismo nivel de ingresos, el mismo nivel de privación material y la misma intensidad de trabajo y estar en situación diferente por tener un enfermo crónico a su cargo, o un peor acceso a los servicios públicos, como el transporte, la educación o la sanidad. Dos familias pueden coincidir también en los tres indicadores, pero una estar más endeudada que otra. El endeudamiento es una importante causa de la pobreza en la Europa del presente. Basta pensar en los casos de personas desahuciadas por no poder pagar la hipoteca y que quedan debiendo todavía una fuerte suma al banco. Sin embargo, el grado de endeudamiento no es contemplado por ninguno de los tres indicadores de la Estrategia 2020.

Por último, hay que tener en cuenta el impacto que las transferencias sociales (pensiones y subsidios) tienen sobre la tasa de personas pobres. Los datos de Eurostat permiten comparar el nivel de pobreza antes y después de dichas transferencias. Las diferencias entre los distintos Estados son muy grandes. Los que tienen un sistema de bienestar robusto son capaces de disminuir en gran medida la tasa de pobreza. En Dinamarca, los subsidios y las pensiones reducen el número de personas potencialmente pobres a menos de la mitad. En 2016, antes de las transferencias hubiera sido pobre el $25 \%$ de la población. Después, esa tasa se rebajó al $12 \%$. En España hubiera habido una tasa de pobreza del 30\% de la población y las transferencias sociales solo la redujeron en una cuarta parte: al 22\%. En el caso de Italia, las transferencias redujeron la tasa de pobreza únicamente del $26 \%$ al $21 \%{ }^{11}$

\footnotetext{
${ }^{11}$ Los datos sobre la situación antes y después de las transferencias sociales pueden encontrarse en las siguientes páginas de Eurostat:

http://ec.europa.eu/eurostat $/ \mathrm{tgm} /$ table.do?tab=table\&init $=1 \&$ language $=$ en\&pcode $=$ tesov $250 \&$ plugin $=1$ (antes)

http://ec.europa.eu/eurostat $/$ tgm/table.do?tab $=$ table\&init $=1 \&$ language $=$ en\&pcode $=$ tps $00184 \&$ plugin $=1$ (después).
} 
El número de personas que dependen de las transferencias sociales (distintas de las pensiones) para no caer en la pobreza es sorprendentemente alto: un $10 \%$ de la población europea. Esa cantidad equivale a la mitad de quienes dependen de las pensiones. Estamos hablando, pues, en total, de un 30\% de los habitantes de la UE. El porcentaje de personas pobres ha ido aumentando en ambos colectivos (Arriola, 2014). Esto demuestra la insuficiencia de una política de lucha contra la pobreza basada exclusivamente en la creación de empleo. De hecho, una política laboral dirigida a hogares con baja intensidad de trabajo puede disminuir sus ingresos si estos hogares se ven afectados por recortes en los subsidios públicos y los trabajos son precarios o mal pagados.

\section{LA PARTICIPACIÓN DE LOS STAKEHOLDERS}

Dada la importancia de las políticas sociales de un Estado para paliar los efectos de la pobreza y la exclusión social, resulta muy relevante conocer cómo se elaboran los documentos que tratan de dar respuesta a estas situaciones de riesgo de pobreza. Para analizar la participación de los stakeholders en este ámbito se ha realizado un trabajo de análisis teórico y empírico. Por un lado, se han estudiado los propios documentos españoles sobre el Semestre Europeo. Por el otro, se han realizado diversas reuniones y entrevistas: con miembros del Ministerio de Sanidad, Servicios Sociales e Igualdad (2016); con la Red Europea Contra la Pobreza (2016 y 2017) y con la Taula d'Entitats del Tercer Sector de Catalunya (2016). También se ha obtenido información a través de un cuestionario respondido por el equipo económico de una eurodiputada española (2016). El objetivo era tratar de descubrir en qué medida los stakeholders han participado en el diseño e implementación de las políticas contra la pobreza y la exclusión social (especialmente en el caso español, aunque se han presentado cuestionarios en diversos países de la Unión Europea).

Para establecer el grado de participación, se han definido cuatro niveles de intensidad. El primer nivel consistiría en recibir información. El segundo, ser consultados. El tercer nivel de participación se daría en caso de que los stakeholders tomaran parte en los procesos decisorios, teniendo voz y voto en los organismos que tienen la potestad de tomar decisiones. El cuarto y último nivel de intensidad es el empoderamiento, que implica tener la capacidad de implantar las políticas, lo que supone no solamente decidir sobre cómo van a ser, sino llevarlas a cabo.

A nivel europeo, el órgano más importante respecto a las políticas sociales es el Comité de Protección Social. Sin embargo, las ONGs no participan en sus sesiones. Solo asisten los representantes de los Estados. Se trata, además, de reuniones opacas: no hay registros, no hay actas, y no se hace público el orden del día. Por ese lado podemos decir que la participación es nula. Las pretensiones de legitimidad no se cumplen ni a su más bajo nivel.

En cuanto a los documentos, los Programas Nacionales de Reforma (PNR 2015, 2016, 2017) son los más importantes a nivel estatal. Recogen los objetivos de actuación de los Estados en el marco del Semestre Europeo, y deben presentarse anualmente de forma obligatoria. Abarcan determinadas políticas públicas, incluidas 
las políticas sociales; están comprendidas, por tanto, las políticas de lucha contra la pobreza.

Los organismos europeos han insistido en la necesidad de participación por parte de las organizaciones sociales en estos documentos. Desde 2014 se han impulsado iniciativas para garantizar un diálogo real con entidades del Tercer Sector, tanto a nivel estatal como europeo. Con ello se ha pretendido garantizar una participación (en sentido amplio; en ocasiones es una mera información) por parte de los interesados o stakeholders (Susías, C.; Malgesini, G., comunicación personal, 7 de junio de 2016).

Según fuentes del Ministerio de Sanidad, Servicios Sociales e Igualdad (MSSSI) español, la elaboración de los PNR depende en última instancia de la Oficina Económica de Presidencia del Gobierno, que mantiene un diálogo directo con las entidades del Tercer Sector. En marzo de cada año, la Oficina envía a la Plataforma del Tercer Sector ${ }^{12}$ un documento para que se realicen propuestas para ser incluidas en el PNR, y se celebran una o dos reuniones formales con la Oficina Económica. Este diálogo resulta de importancia porque, para las Entidades Sociales, lograr introducir un tema concreto en un PNR implica que la Comisión pueda cuestionar al Estado sobre el mismo. Es una forma de lograr que un problema determinado no sea sistemáticamente ignorado.

Los propios Planes Nacionales de Reforma hacen referencia a esta participación en sentido amplio. El PNR de 2015 establece que el Tercer Sector tuvo «un papel muy activo» en la elaboración del documento, y recoge las aportaciones realizadas por determinadas entidades como el Tercer Sector de Acción Social o la Plataforma del Tercer Sector (PNR, 2015: 92-93).

El PNR de 2016 recoge fórmulas similares, aunque es considerablemente más escueto debido a que fue elaborado por un gobierno en funciones. Según EAPN, se trata de un PNR más valorativo sobre las actuaciones llevadas a cabo hasta el momento, pero que no va más allá de lo que la UE exigía para el Plan de Estabilidad (C. Susías; G. Malgesini, 2016).

El PNR de 2017, por su parte, recoge incluso una menor participación por parte del Tercer Sector que la versión de 2015. Lo cierto es que, al margen de las consideraciones sobre los niveles de participación real y efectiva, puede apreciarse una reducción de la participación por parte de las entidades entre 2015 y 2017 en función de lo que recogen los propios PNR.

En relación con los niveles de participación previamente definidos, puede afirmarse que en la elaboración de los PNR en España existe una participación en los dos primeros niveles. En este sentido, las entidades (al menos, algunas de ellas) reciben información por parte de la Oficina Económica de Presidencia del Go-

\footnotetext{
12 La Plataforma del Tercer Sector reúne siete organizaciones de las más representativas del ámbito de lo social a nivel español: la Plataforma del Voluntariado de España (PVE), la Red Europea contra la Pobreza y la Exclusión Social del Estado Español (EAPN España), la Plataforma de ONG de Acción Social (POAS), el Comité Español de Representantes de Personas con Discapacidad (CERMI), Cruz Roja Española, Cáritas y la Organización Nacional de Ciegos de España (ONCE).
} 
bierno, mantienen reuniones formales e incluso son consultados, pues realizan propuestas para que sean incluidas en el documento (niveles 1 y 2).

La participación a estos dos primeros niveles es, por tanto, elevada. El Gobierno tiene la obligación de preguntar a la sociedad civil, lo que incluye consultas a sindicatos, y asociaciones de empresarios, así como entidades del Tercer Sector. Organizaciones como EAPN tienen una interacción directa con la Oficina Económica, que es en definitiva quien cierra el PNR. En marzo, la Oficina envía un documento a la Plataforma del Tercer Sector solicitando las propuestas que esta quiere hacer constar en el PNR, y antes de contestar se celebran una o dos reuniones formales. Finalmente, la Oficina envía a las entidades el PNR el mismo día que lo hace público, y las entidades confeccionan un documento para valorar el impacto de las aportaciones del Tercer Sector en la versión final del PNR. Este documento también se hace público, y se envía a la UE y a la Oficina del Semestre.

En el ámbito español, destaca la Comisión para el Diálogo Civil con la Plataforma del Tercer Sector, que pertenece al MSSSI y que, formalmente, preside el Secretario o Secretaria de Estado, aunque en diversas ocasiones participa el Ministro o Ministra titular. Se trata de una Comisión con un papel claro de interlocución con el Gobierno, por ejemplo, a la hora de fomentar que se inicie un plan de atención específico. Anualmente se realiza una reunión con el Ministro/a y todos los Directores/as Generales para explicar a la Plataforma del Tercer Sector las políticas que se van a llevar a cabo durante el año siguiente, y la Plataforma puede acudir a la reunión con distintos temas que considere oportuno tratar con el Ministerio. Estas reuniones son de carácter formal y político, por lo que paralelamente se mantienen otras de tipo técnico con las direcciones generales concretas o con otros ministerios, en las que siempre hay un representante del Ministerio de Hacienda presente (Susías, C.; Malgesini, G., 2016).

Junto a la Comisión para el Diálogo Civil destaca el Consejo Estatal de ONG de Acción Social, órgano consultivo adscrito al MSSSI. Este órgano se articula en torno a Grupos de Trabajo, y en él las entidades se encuentran representadas en función de su ámbito de actuación. Se encarga del seguimiento y negociación de determinadas estrategias con el ministerio correspondiente. Al estar articulado en torno a grupos de trabajo, cada grupo interviene cuando el plan a desarrollar pertenece a su propio ámbito de actuación (ej. Infancia).

Se trata de un proceso de participación amplio y de funcionamiento democrático, aunque los resultados no son satisfactorios; desde entidades como la EAPN se señala que los planes de actuación o las iniciativas raramente son aprobados con una memoria económica que los haga posibles (de ahí la importancia del representante del Ministerio de Hacienda en las reuniones). En este sentido, se aprueban iniciativas que no pueden ser efectivamente desarrolladas porque no existe dotación económica para ello. Por este motivo son muchas las entidades que cuestionan su eficacia, si bien consideran que son espacios necesarios (Susías, C.; Malgesini, G., 2016).

También se han encontrado trabas a la hora de tomar la decisión de incluir o no determinados temas. EAPN pretendía incluir en el PNR de 2016 la respuesta del Gobierno a los denominados Informes Específicos sobre el País, elaborados 
por los servicios de la Comisión en el marco del Semestre Europeo. La Oficina de Presidencia se negó, y elaboró un PNR que daba respuesta únicamente a las Recomendaciones Específicas y no al Informe de País, documento que de alguna forma complementa las Recomendaciones (Susías, C.; Malgesini, G. 2016). Por lo demás, desde EAPN se considera que la elaboración de los PNR en España toma en consideración las aportaciones de las entidades sociales de forma más intensa que en otros países (Susías, C., comunicación personal, 4 de julio de 2017). Es decir, existe una buena participación de primer nivel (recibir información) y de segundo nivel (consulta), si bien la última palabra la tiene el Gobierno. Las entidades no tienen poder de decisión final para incorporar cualquier aspecto a los PNR, y mucho menos para implementar las políticas determinadas en estos documentos.

En términos de información y consulta, por tanto, la valoración por parte de la EAPN es positiva. En España, el Tercer Sector está fuertemente organizado y articulado. En este sentido, por tanto, los mecanismos de gobernanza respecto a políticas sociales son percibidos como "muy positivos" en cuanto a las posibilidades de participación. No obstante, esta opinión difiere de lo expresado por la Taula del Tercer Sector (que participa en EAPN España). Según su director general, la incidencia de la Taula del Tercer Sector en la elaboración de documentos es nula. A través de su participación en EAPN están informados, pero sin posibilidades de incidir. Por eso la valoración de la Taula del Tercer Sector respecto a la participación difiere considerablemente de lo expresado por EAPN (Codina, T., comunicación personal, 29 de junio de 2016). Para la Taula del Tercer Sector, su participación se limitaría al primer nivel (información).

A pesar de todo, también para la EAPN parece ser que esta participación tiene escasos efectos reales. A nivel europeo, el marco de la estrategia Europa 2020 y el Semestre Europeo ofrecen a las entidades una oportunidad relevante para manifestar sus posturas e incidir en políticas estatales, pero que en última instancia no obliga a los Estados. Resulta útil, en este sentido, en términos de poner en la agenda pública determinados problemas, tratando de obligar a los partidos políticos y a los Estados en general a abordar un problema concreto. EAPN reconoce así que la participación se limita a los dos primeros niveles, pues ni tienen poder decisorio (no pueden incorporar a los PNR, y por tanto a las políticas públicas estatales, los aspectos que ellos como stakeholders consideren oportunos) ni juegan papel alguno en la implementación de las políticas, ámbito que queda estrictamente delegado a los poderes públicos. Esta parece ser la tónica general en todos los países analizados, pues en todos ellos existe información a las entidades sociales, y en la mayoría también consulta. Sin embargo, ningún país tiene incidencia real en la toma e implementación de decisiones, salvo una única excepción: Holanda (EAPN, 2017).

\section{CONCLUSIÓN}

La gobernanza es una nueva forma de dirección de las políticas de interés general. Se caracteriza por permitir la participación de actores privados en su diseño, por priorizar la coordinación negociada sobre la jerarquía en la toma de decisiones y 
por utilizar sistemas de incentivos en lugar de sanciones. La Unión Europea es un terreno especialmente fecundo en formas de gobernanza. El experimentalismo democrático es una de ellas. Sus teóricos ponen un énfasis especial en la participación de los interesados en procesos deliberativos como mecanismo legitimador para la adopción de decisiones. Esta especie de gobernanza es la que se utiliza en la UE para coordinar las políticas sociales en general y las políticas contra la pobreza en particular.

La realidad de la pobreza en Europa es un fenómeno innegable que afecta a un gran porcentaje de la población, aunque la situación difiere mucho de unos Estados a otros. El año 2010, la UE aprobó la estrategia Europa 2020, uno de cuyos objetivos era sacar a veinte millones de personas de la pobreza durante la presente década. Sin embargo, el número de pobres no solo no se ha reducido, sino que ha aumentado desde entonces. La causa principal de este incremento han sido las medidas de austeridad adoptadas como consecuencia de la crisis de la Eurozona. Las políticas de lucha contra la pobreza, responsabilidad de los Estados, han carecido de la fuerza suficiente como para contrarrestar los efectos sociales de unas políticas económicas impuestas desde la Unión.

Por otro lado, la estrategia de lucha contra la pobreza impulsada desde Europa no ha sido la adecuada. Se ha puesto el énfasis casi exclusivamente en la creación de empleo, sin tener en cuenta que puede haber otras causas de la pobreza, además del paro y, sobre todo, sin garantizar formas de trabajo digno. Como consecuencia, muchas personas que han encontrado empleo no han logrado con ello dejar de ser pobres. Otras han visto deteriorarse su situación como consecuencia de la mengua en las transferencias sociales, como las pensiones o los subsidios. Y todos los usuarios de los servicios públicos han perdido capacidad de acceso a los mismos debido a los recortes presupuestarios en políticas sociales como la educación o la sanidad. La única forma de luchar eficazmente contra la pobreza en Europa sería un cambio radical en las políticas económicas y financieras de los Estados.

El análisis de la participación de las Entidades del Tercer Sector en la definición e implementación de las políticas sociales resulta fundamental para valorar la legitimidad de los mecanismos de gobernanza utilizados en el marco del Semestre Europeo, que plantea unos objetivos ambiciosos de cara a reducir la cantidad de personas en riesgo de pobreza y exclusión social. En base a los cuatro niveles de participación descritos, se puede afirmar que dicha participación es realmente escasa. El Gobierno de España mantiene relaciones constantes con estas entidades, a las que informa de los plazos y procesos de la elaboración e implementación de políticas (nivel 1). También consulta a estas entidades su opinión sobre dichas medidas, de forma que aquellas puedan proponer cambios o políticas concretas (nivel 2). Sin embargo, esto último no ocurre con todas las entidades; algunas de ellas solo reciben información a través de EAPN o la Plataforma del Tercer Sector, y no participan en modo alguno en la elaboración de propuestas. Así, la interacción entre el Gobierno y las entidades parece querer legitimar los mecanismos de gobernanza utilizados en el marco del Semestre Europeo, pero es posible poner en duda 
que los stakeholders tengan realmente capacidad para incidir en las políticas que se llevan a cabo.

En este sentido, ni siquiera EAPN, que parece ser la entidad con mayores canales de interacción con el Gobierno, puede tomar decisiones acerca de las políticas contra la pobreza y la exclusión social que se llevan a cabo (nivel 3). Tan solo puede proponer, pero la última palabra depende del Gobierno. Por supuesto, no cuentan con mecanismo alguno para implementar las políticas adoptadas (nivel 4), por lo que la participación real parece ser escasa. La misma EAPN así lo reconoce; aunque valoran positivamente el incremento de participación en los dos primeros niveles, reconocen que sus propuestas quedan a merced de otros factores relevantes y que escapan a su intervención, como las decisiones del Ministerio de Hacienda, que no suele dotar de contenido económico a las medidas adoptadas, o los requisitos presupuestarios que la Unión Europea exige a los Estados Miembros. Por ello, cabe poner en cuestión que los mecanismos de gobernanza adoptados se encuentren legitimados por la efectiva participación de los interesados o stakeholders. Y, a juzgar por el incremento de personas en riesgo de pobreza o exclusión social, tampoco por los resultados.

\section{GLOSARIO DE SIGLAS}

CPS: Comité de Protección Social

EAPN: European Anti Poverty Network (sección española: Red Europea de Lucha contra la Pobreza y la Exclusión Social en el Estado Español).

MSSSI: Ministerio de Sanidad, Servicios Sociales e Igualdad

PNR: Programa Nacional de Reforma

PTSA: Plataforma del Tercer Sector de Acción Social

UE: Unión Europea

\section{REFERENCIAS BIBLIOGRÁFICAS}

Textos

Arriola, J. (2014). La estrategia de la Unión Europea de lucha contra la pobreza, VII informe sobre exclusión y desarrollo social en España 2014. Documento de trabajo 8.2.

Copeland, P. et al. (2014). "Poverty and social policy in Europe 2020: ungovernable and ungoverned", Policy \& Politics. United Kingdom: Policy Press, 42(3), pp. 351-365.

Crozier, M., Huntington, S. P. and Watanuki, J. (1975). The crisis of democracy. New York: New York University Press 
EAPN (2014). Poverty and Inequality in the EU.

EAPN (2017). Social Rights or Social Plight? | EAPN Assessment of the 2017 National Reform Programmes.

Estévez Araujo, J. A. (2006). El revés del derecho, Bogotá: Universidad Externado de Colombia.

Mercado Pacheco, P. (2012). "Experimentalismo democrático, nuevas formas de regulación y legitimación del Derecho", Anales de la Cátedra Francisco Suárez, 46, Granada: Universidad de Granada, pp. 37-68.

Peña-Casas, R. (2012). "Europe 2020 and the fight against poverty and social exclusion: fooled into marriage?", Social Developments in the European Union 2011: Thirteenth Annual Report. ETUI, p. 159.

Prats Català, J. (2005). De la burocracia al management, del management a la gobernanza : las transformaciones de las administraciones públicas de nuestro tiempo. Madrid: Instituto Nacional de Administración Pública. Available at: http://ccuc.cbuc.cat/record=b3447610 S23*cat (Accessed: 27 April 2016).

\section{Documentos oficiales}

PNR. Programa Nacional de Reformas del Reino España 2015: https:/ / ec.europa.eu/info/files/2015-european-semester-national-reformprogramme-spain_es

- Programa Nacional de Reformas del Reino de España 2016: https://ec.europa.eu/info/sites/info/files/european-semester-nationalplan_spain_2016_es.pdf

- Programa Nacional de Reformas del Reino de España 2017: https://ec.europa.eu/info/sites/info/files/2017-european-semesternational-reform-programme-spain-es.pdf 